\title{
Corela
}

Cognition, représentation, langage

HS-7 | 2010

Espace, Préposition, Cognition

\section{Les adverbiaux prépositionnels en dans : exploration en corpus de la notion de contenance}

Laure Sarda

\section{OpenEdition}

\section{Journals}

Édition électronique

URL : http://journals.openedition.org/corela/911

DOI : 10.4000/corela.911

ISSN : 1638-573X

\section{Éditeur}

Cercle linguistique du Centre et de I'Ouest - CerLICO

Référence électronique

Laure Sarda, «Les adverbiaux prépositionnels en dans : exploration en corpus de la notion de contenance », Corela [En ligne], HS-7 | 2010, mis en ligne le 31 mai 2010, consulté le 20 avril 2019. URL : http://journals.openedition.org/corela/911 ; DOI : 10.4000/corela.911

Ce document a été généré automatiquement le 20 avril 2019

\section{(c) (1) (2)}

Corela - cognition, représentation, langage est mis à disposition selon les termes de la licence Creative Commons Attribution - Pas d'Utilisation Commerciale - Partage dans les Mêmes Conditions 4.0 International. 


\title{
Les adverbiaux prépositionnels en dans : exploration en corpus de la notion de contenance
}

\author{
Laure Sarda
}

1 Vandeloise s'est longuement appliqué dans son œuvre à critiquer ardemment la description des relations spatiales exprimées dans la langue par les outils, inadéquats, de la géométrie. En ce qui concerne la préposition dans, il a montré qu'elle ne peut être définie par l'inclusion topologique sans fatalement mener à «une ontologie fantomatique ». Il remarque non sans humour :

«s'il est vrai que je suis dans mon fauteuil, je n'y suis pas inclus topologiquement

sans quoi, n'étant pas une pure extension, je risquerai de passer à travers "

(Vandeloise 2001 : 234).

2 Les dix pages qui précèdent cet extrait sont une démonstration de plus ${ }^{1}$ de l'inadéquation des concepts purement géométriques pour décrire la sémantique de dans. Nous ne reviendrons pas sur cet aspect que nous considérons comme acquis, pour nous consacrer dans cet article à une approche fonctionnelle qu'il qualifie en 2001 de dynamique.

3 Nous nous pencherons sur la définition de la relation de contenance et ses extensions (dépendance et zone d'influence). Notre terrain d'observation sera cependant bien différent de celui de Vandeloise qui a précisément décrit la préposition dans dans des structures élémentaires $x$ est dans $y /$ il y $a x$ dans $y$ où $x$ désigne la cible (entité à localiser) et $y$ le site (entité localisatrice), sur des exemples forgés à des fins illustratives. Nous nous placerons sur le terrain de l'usage (étude sur corpus Le Monde) et nous observerons en particulier les syntagmes prépositionnels introduits par dans en position initiale de phrase, dans la construction [Dans SN, P]. Pourquoi ? Premièrement pour modestement restreindre notre étude, afin de mieux la cerner. Deuxièmement, parce que dans cette position initiale précisément, le site (Dans y) est posé avant la cible qu'il va falloir identifier. Libéré du domaine étroit de la prédication, la position initiale, périphérique, offre une large gamme de possibilités de portée ou de rattachements. Nous esquissons dans cet article une description des sens notionnels de la construction [Dans SN, P] où le 
site $y$ est dénoté par le SP extraprédicatif [Dans $\mathrm{SN}$ ] et la cible par tout ou partie de la phrase P, phrase d'accueil du SP détaché.

4 A partir de l'observation de la diversité des usages, nous défendrons l'idée que la forme dans est une unité polysémique dont les différentes facettes s'éclairent au contact des unités qu'elle connecte. Nous suivrons totalement Vandeloise dans sa recherche méthodologique qui consiste à saisir cette variation non pas dans une forme abstraite au pouvoir descriptif nécessairement limité, mais par un faisceau de traits organisés en ressemblance de famille.

5 Partant de cette organisation en ressemblance de famille, nous acceptons également l'idée (controversée - cf. Franckel \& Paillard 2007) que dans est une préposition spatiale dans ses usages prototypiques. Nous défendons la thèse localiste non pas tant en synchronie mais dans une vision dynamique d'évolution des langues (cf. Fagard \& Sarda, 2009, Heine \& al. 1991, Hopper \& Traugott 1993, Tyler \& Evans 2003, Evans \& Tyler 2004) et dans le sens beaucoup plus profond où notre rapport expérienciel à l'espace détermine notre capacité à structurer nos pensées «notre espace mental» de façon analogique à l'espace physique (Lakoff 1987, Lakoff \& Jonhson 1999, Stewart 2001).

\section{La notion de contenance}

6 La relation contenant/ contenu $(\mathrm{C} / \mathrm{c})$ est une relation extra-linguistique décrite par une liste de traits, structurée comme une ressemblance de famille. Vandeloise la définit par un ensemble de cinq traits qui, lorsqu'ils sont tous réunis, décrivent une relation $\mathrm{C} / \mathrm{c}$ prototypique. Cependant, aucune de ces propriétés n'est une condition nécessaire et suffisante de l'existence de la relation. Ces traits sont les suivants (cf. Vandeloise 2001: 239) :

(a) Le contenant contrôle la position du contenu

(b) S'il y a mouvement, le contenu se déplace vers le contenant

(c) Le contenant entoure le contenu ${ }^{2}$

(d) Le contenant protège le contenu

(e) Le contenant cache le contenu

7 Vandeloise note que cette liste varie peu à travers le monde et que chaque langue « choisit un certain nombre de traits qui lui permettent d'étendre l'usage du mot associé à la relation $\mathrm{C} / \mathrm{c}$ prototypique à des manifestations marginales de cette relation " (idem :240). Selon lui, le français ne fait pas appel au trait (e) pour étendre l'usage de la préposition dans ${ }^{3}$. Tyler \& Evans (2003) montrent en revanche que in en anglais développe un ensemble d'usages (qu'elles désigne par The Disappearance Sense) qui repose sur ce trait et caractérise les emplois comme The wine quickly soaked in, Millie rubbed in the lotion, ou The sun has gone in./ The sun is in (Tyler \& Evans 2003 : 195).

Dans les couples d'exemples qui suivent (que nous reprenons à Vandeloise 1986 \& 2001) les traits (a-d) sont respectivement satisfaits par le premier exemple et non satisfaits par le second.

(1) a. La lampe est dans la douille/ le doigt est dans la bague

b. La mouche est dans le bol/ la soupe est dans la louche

c. Le vin est dans le verre/ Les fleurs sont dans le vase

d. Le cadeau est dans l'emballage/ l'enfant est dans la rue

Pour le trait (a), la douille contrôle bien la position de l'ampoule mais à l'inverse, c'est le doigt qui contrôle la position de la bague. De ce fait, ce dernier exemple est moins 
prototypique que le premier eu égard au trait (a). De même pour le trait (b) la soupe est dans la louche est moins typique que la mouche est dans le bol car c'est la louche qui bouge vers la soupe plutôt que l'inverse etc.

En associant cette relation $\mathrm{C} / \mathrm{c}$ à la préposition dans et par force démonstrations, Vandeloise a établi que l'inclusion topologique n'est pas une condition nécessaire à l'usage de la préposition dans. Il s'est appliqué à montrer qu'elle n'est pas non plus une relation suffisante au travers de l'étude des relations partie-tout. Il passe en revue les rapports lieu/région (Athènes et l'Attique), membre / collection (Les arbres et la forêt), composant/ assemblage (l'anse et la tasse), matière/objet (le marbre et le temple), portion/ masse (la tranche de melon et le melon) et membre/ classe (la rose et les fleurs). Il examine attentivement le type d'entités pouvant être mises en relation par dans pour finalement repousser la dichotomie entre entités matérielles et entités spatiales.

Plutôt que la distinction matériel/spatial, ce sont les concepts de dépendance matérielle et de zone d'influence qui permettront de traiter les usages de la préposition dans mal expliqués précédemment ${ }^{4}$. Ces deux concepts peuvent être considérés comme des développements de la relation $\mathrm{C} / \mathrm{c}$, rendus nécessaires, notamment, par la nature des entités spatiales. Ils pourraient ouvrir la voie à une étude systématique des emplois métaphoriques de la préposition dans (Vandeloise 2001 : 256-257).

Le concept de dépendence matérielle rapproche des cas impliquant la mise en relation d'une cible matérielle ou spatiale. Qu'il s'agisse du trou (entité spatiale) dans la table, ou du noeud (entité matérielle) dans la corde, dans les deux cas, l'existence du trou et du nœud dépendent matériellement de la table et de la corde, respectivement. La cible dépend du site non seulement pour sa localisation mais aussi pour son existence-même. La règle est formulée comme suit :

$a$ est dans $b$ si l'existence de a dépend matériellement de $b$

Quant au concept de zone d'influence, il permet de rendre compte des cas du type les armes sont interdites dans ce périmètre; le navire est dans le triangle des Bermudes; il n'y a pas d'arbre dans un rayon de cent kilomètres (cf idem :261). Pour ces cas, Vandeloise avance que le locuteur attribue des propriétés spécifiques à la zone délimitée, sans quoi cette délimination n'aurait aucune raison d'être. En conséquence, les cibles sont affectées par les entités spatiales dans lesquelles elles se trouvent. La règle est ainsi formulée :

a est dans une entité spatiale b si a subit les conséquences de la zone d'influence associée à b

Suivant la voie ouverte par Vandeloise, nous tentons d'explorer dans la section 3.2. comment ces deux concepts permettent d'étendre la description aux emplois métaphoriques. Nous avons exposé dans cette première section un compte rendu rapide de la relation extra-linguistique $\mathrm{C} / \mathrm{c}$ et de son extension par les concepts de dépendance matérielle et de zone d'influence. Nous avons rapidement illustré en reprenant les exemples de Vandeloise les types d'emplois de la préposition dans qu'elle permet d'expliquer. Une conception du sens est ainsi posée qui résulte de l'interaction de l'homme avec son environnement. Une conception qui s'écarte largement des conceptions structuralistes pour motiver les sens par des facteurs extra-linguistiques, des facteurs fonctionnels (pour une description détaillée, voir Vandeloise 1986). 


\section{Les emplois de dans en corpus}

Nous commencerons en section 2.1 par présenter des résultats quantitatifs qui offrent un panorama de la distribution des sens de dans. Nous examinerons en 2.2., d'un point de vue plus qualitatif, les emplois notionnels (métaphoriques ou abstraits) lorsqu'ils apparaissent en tête et tenterons de les confronter aux différents traits de la ressemblance de famille de la relation $\mathrm{C} / \mathrm{c}$ en 2.3.

\subsection{Radioscopie des sens de dans en corpus}

Les résultats présentés ici sont tirés d'une étude de 3000 occurrences de dans extraites automatiquement de LeMonde (dec 2000) à l'aide de SYNTEX ${ }^{5}$. Les données annotées sont extraites d'une base de données d'analyse des syntagmes prépositionnels, réalisée dans le cadre du projet Spatial Framing Adverbials ${ }^{6}$. Nous avons sélectionné pour la présente étude les SP en dans dans les positions initiale, médiane et finale, liées à leur fonction : (i) en tête et non régis; (ii) en zone post-verbale et régis par un constituant verbal (verbe conjugué, verbe à l'infinitif ou verbe participe $)^{7}$; et (iii) en queue non régis ${ }^{8}$. Ces trois zones sont illustrées respectivement par les exemples (2-4) ci-dessous :

(2) Tête: [* <* Dans *> les pays démocratiques *], quand un gouvernement considère qu'un article est calomnieux, il porte l'affaire devant les tribunaux..

(3) Régis par V : L'une, justement, $\left\{{ }^{*}\right.$ passe $\left.{ }^{*}\right\}$ timidement une tête $\left[{ }^{*}<*\right.$ dans ${ }^{*}>$ son bureau*], sans frapper.

(4) Queue : La présidence française de l'Union européenne a fait l'objet de critiques, parfois violentes, $\left[*{ }^{*}\right.$ dans $*>$ plusieurs capitales européennes $*$ ].

Les emplois de dans sur ces trois zones se répartissent pour quasi $50 \%$ sous l'influence verbale, et l'autre moitié sur les zones périphériques avec, en gros, un tiers en queue et deux tiers en tête comme le montre la figure 1.

Figure 1

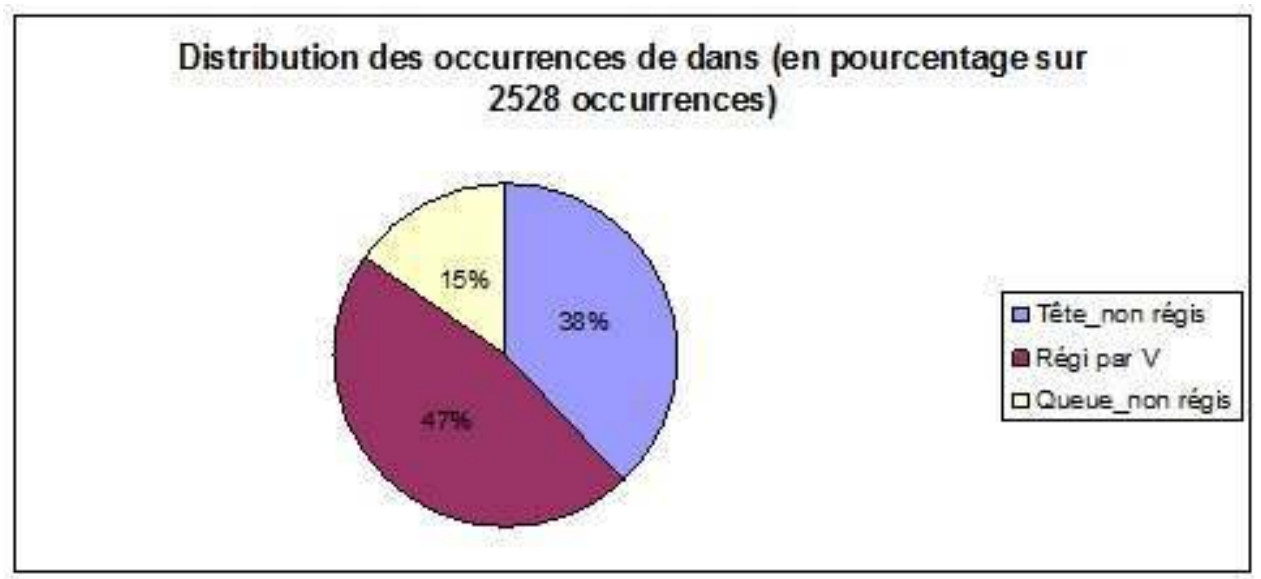

17 Notons que sur les 3000 occurrences initiales, près de 470 ont été éliminées dans la zone postverbale parce qu'elles n'étaient ni régies par un constituant verbal, ni non régies. Ont été écartés (pour la présentation des résultats dans cet article) les cas où le SP en dans était régi par un nom, un adverbe, un adjectif, une coordination, etc, à l'exception d'un constituant verbal. 
Sur cette base, nous avons observés la répartition des primitives de dans. Ces primitives ont été définies dans le cadre du projet SFA pour décrire le sens de neuf prépositions différentes. Elles correspondent aux grandes catégories cognitives que sont : l'espace, le temps, le notionnel (qui englobe les emplois métaphoriques et les emplois abstraits), la cause. Nous avons ajouté une cinquième primitive "énonciatif " pour capter tous les emplois qui relèvent de l'énonciation. Ces cinq primitives sont illustrées ci-dessous par des usages en tête :

(5) Espace : [ ${ }^{*}$ Dans * $>$ le vieux Caire *], il faut se baisser pour entrer dans l'église d'Abu Serga (Saint-Serge).

(6) Temps : [* <* Dans *> les années 50 *], quelque deux mille malchanceuses mouraient, chaque année, des suites d'un avortement clandestin.

(7) Notionnel : [ ${ }^{*}{ }^{*}$ Dans ${ }^{*}>$ un réalisme prenant $*$, la troupe porte collectivement la pièce vers le conte pour adultes : il était une fois un roi (boucher de son état) et ses trois fils.

(8) Cause : [* ${ }^{*}$ Dans * $>$ la crainte de représailles *], non seulement l'ONU, mais aussi les organisations non gouvernementales, ont déjà procédé à l'évacuation de leur personnel humanitaire en Afghanistan.

(9) Enonciatif: [* $<*$ Dans * $>$ la meilleure tradition de l'unanimisme cher aux Américains *], il a retourné à Al Gore l'hommage que celui-ci venait de lui rendre, confirmant que les deux hommes allaient se rencontrer mardi prochain à Washington.

19 Les emplois intrinsèquement causatifs et énonciatifs sont marginaux avec la préposition dans. Leur faible représentation ne ressort pas en pourcentages. On constate par ailleurs que les emplois de dans sont à $67 \%$ notionnels, $26 \%$ spatiaux et $7 \%$ temporels, toutes positions confondues. En regardant leur répartition selon leur position (figure 2), on voit qu'il n'y a pas de différence nette d'une position à l'autre. Nous avons par exemple presque autant de notionnels dans les trois positions. On note cependant, que les emplois temporels sont deux fois plus nombreux en tête (10\%) que dans les deux autres positions (5\%).

Figure 2

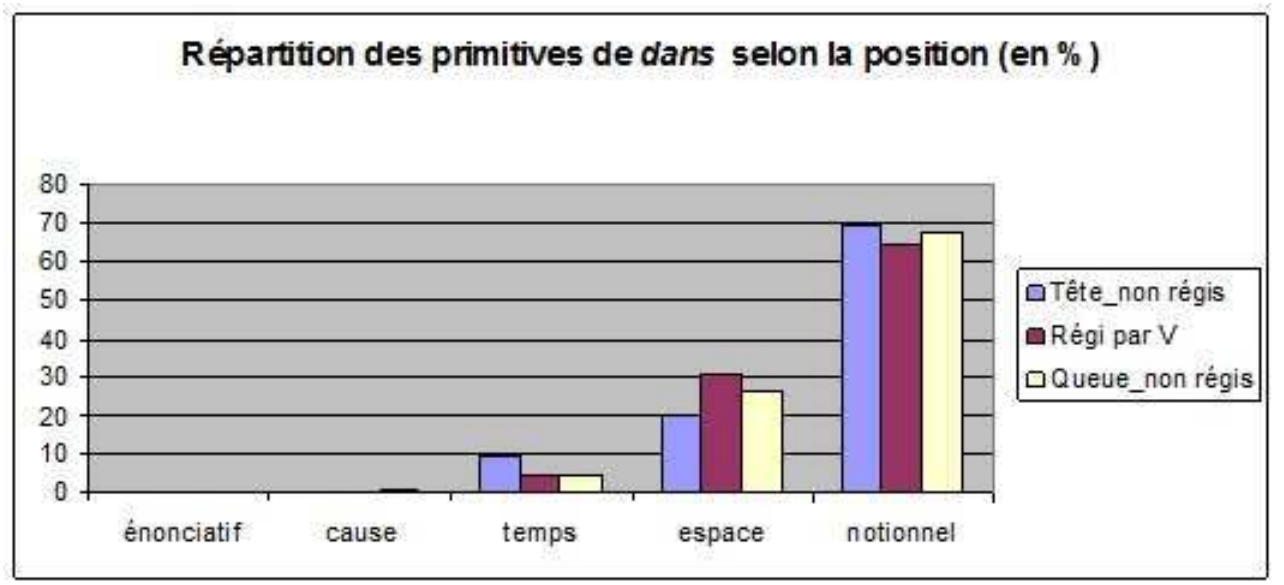

Ces primitives ont le mérite d'avoir permis de constituer des réservoirs de paquets d'exemples exploitables pour l'analyse. Mais leur pouvoir descriptif est limité. Aussi nous avons combiné ces primitives avec des valeurs sémantiques qui permettent de les préciser (et d'interroger la base à un niveau de granularité plus fin). Ces valeurs, au nombre de quinze, ont elles aussi été définies pour pouvoir décrire les 9 prépositions. Elles ne sont 
donc pas toutes pertinentes pour décrire dans. La figure 3 donne une vue d'ensemble de la répartition et de la fréquence des valeurs sélectionnées par dans.

Figure 3

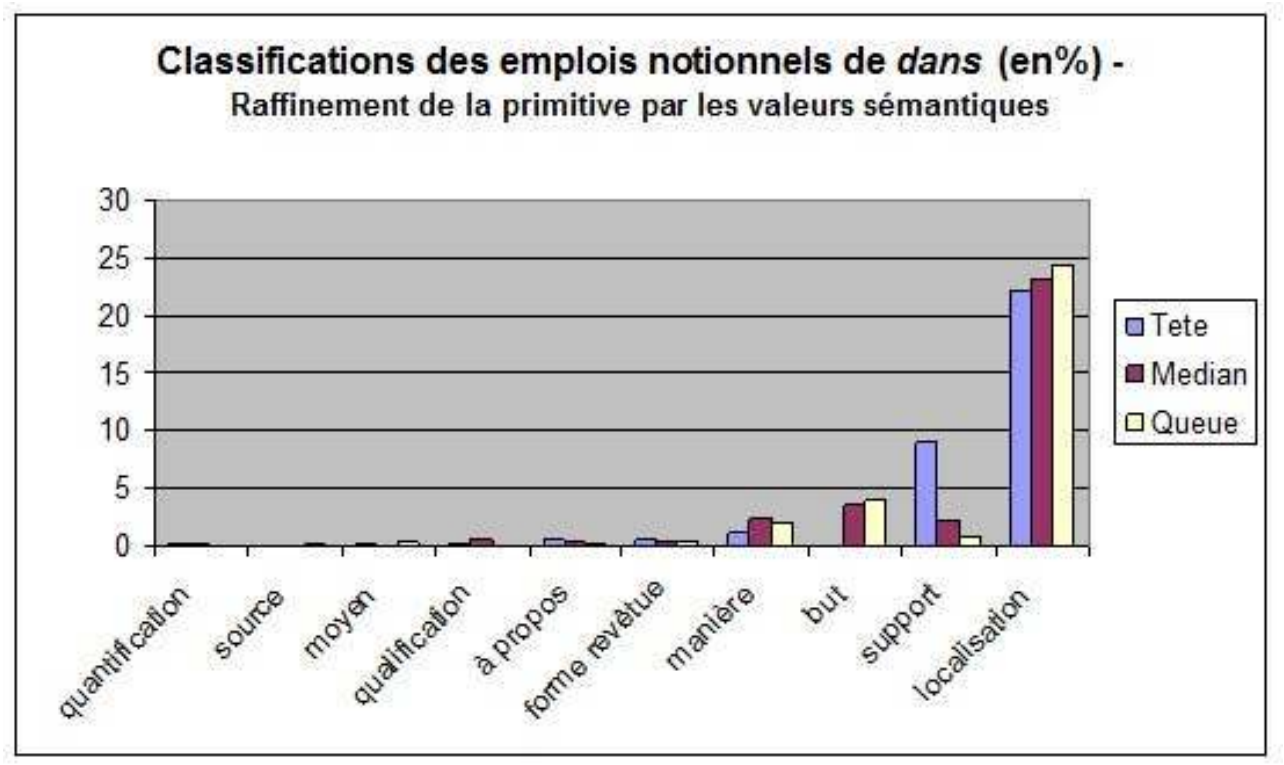

Les emplois notionnels_localisation sont largement majoritaires dans les trois positions (10). Les emplois notionnels_support (11) viennent en deuxième rang de fréquence et sont surreprésentés en tête. Très proches des notionnels_localisation, ils sont spécialisés dans la localisation du " dit ", à travers son support ${ }^{9}$. Les emplois notionnels_but (12) arrivent en troisième rang mais n'apparaissent jamais en tête. La proportion des emplois notionnels_manière en quatrième rang est minime (13). Les autres valeurs, quantitativement marginales, sont illustrées dans les exemples (14-20). Dans tous les cas, l'option (autre) était à la disposition du codeur si les valeurs définies ne lui permettaient pas de caractériser l'exemple à annoter. (14) illustre un cas qui n'avait pas été caractérisé et reste simplement « notionnel ».

(10) localisation : [* <* Dans *> le contexte des turbulences boursières *], personne n'espérait un tel succès.

(11) Support: [* <* Dans *> son bulletin mensuel de décembre, publié *] mardi, la Banque de France juge souhaitable que les engagements des banques françaises dans les télécommunications n'excèdent pas le poids du secteur dans l'économie française et internationale.

(12) but: Le nain se $\{*$ lance $*\}{ }^{*}<*$ dans * ${ }^{*}$ une danse *] où il lutte contre le vent glacé, perd sa robe qui part en lambeaux et offre à Betti un dernier morceau d'étoffe râpée en lui disant que c'est cela le bonheur.

(13) manière : [* ${ }^{*}$ Dans ${ }^{*}>$ une ambiance très arc-en-ciel $\left.{ }^{*}\right]$, mi BD, mi comédie musicale, ce jeune couple découvre l'amour, le goût des pamplemousses, les fêtes à tam-tam.

(14) (autre): [* ${ }^{*}$ Dans * > l'affirmative *], certains n'excluent pas que cette nouvelle réunion pourrait se tenir très rapidement, peut-être dès la semaine prochaine.

(15) forme revêtue ${ }^{10}$ : C'est Daniel Cohn Bendit, [* ${ }^{*}$ dans *> le rôle de M. Loyal *] qu'il affectionne, qui a engagé, mercredi 30 novembre ses amis à soutenir la démarche du premier ministre.

(16) à propos: [* <* Dans *> les champagnes millésimés *]-les meilleures années uniquement-, aucun vin antérieur à l'année de récolte ne peut être ajouté.

(17) qualification : [* ${ }^{*}$ Dans *> son sens étymologique *], la liberté renvoyait à une 
puissance de connexion, d'agrégation, de mise en commun : à une racine commune qui se développe et croît selon sa loi interne.

(18) moyen : [* <* Dans * un arrêt de 1997 *], la Cour de Berlin avait prétendu que les Témoins de Jehovah (170 000 en Allemagne) ne pouvaient prétendre au statut de collectivité de droit public, dont jouissent les Eglises (avec avantages financiers), jugeant qu'ils ...

(19) source : En revisitant l'école de Paris entre 1904 et 1929, le Musée d'art moderne de la Ville de Paris ne $\{*$ célèbre *\} pas seulement la part prise par les «étrangers » $\left[*{ }^{*}\right.$ dans $*>$ les avant-gardes $*$.

(20) Quantification: [* $<^{*}$ Dans * la proportion des deux tiers *], les demandeuses d'emploi ont un niveau de formation inférieur au baccalauréat.

Si les usages des valeurs de dans illustrées en (14-20) sont peu fréquents, ils n'en sont pas moins familiers que les autres (10-13) et leur compréhension en contexte ne pose pas problème. Leur analyse en revanche est particulièrement délicate pour le linguiste. C'est ce que nous allons examiner dans la section suivante.

\subsection{Analyse des emplois notionnels (tête) par la ressemblance de famille $\mathrm{C} / \mathrm{c}$}

Nous avons constitué un sous corpus (66 occurrences) pour explorer plus en détail les emplois notionnels, en tête, indépendamment de leur représentativité dans le corpus. Nous avons pris les quelques occurrences des valeurs les moins fréquentes et dix exemples sélectionnés aléatoirement pour chacune des valeurs plus fréquentes: (manière, support et localisation) ${ }^{11}$.

Nous avons élaboré une grille d'analyse qui indique pour chaque exemple quels sont la cible et le site, leur nature et la relation (méronomique) qu'ils peuvent entretenir. Une fois identifiés les « objets » de la relation nous avons observé si la relation établie satisfait ou pas les traits de la ressemblance de famille $\mathrm{C} / \mathrm{c}$ ( $\mathrm{C}$ contrôle $\mathrm{c}$, mouvement de $\mathrm{c}$ vers $\mathrm{C}, \mathrm{C}$ entoure $\mathrm{c}, \mathrm{C}$ protège $\mathrm{c}, \mathrm{C}$ cache $\mathrm{c}, \mathrm{c}$ dépend de $\mathrm{C}$, et $\mathrm{c}$ est sous l'influence $\mathrm{de} \mathrm{C}$.).

\subsubsection{Identification des termes de la relation quand dans est en tête}

L'identification des termes de la relation n'est pas aisée. Nous n'abordons pas la question en termes de portée du SP, nous cherchons simplement au premier stade de cette analyse à identifier les objets de la relation et leur nature 'ontologique' pour pouvoir ensuite établir quel type de relation ils entretiennent via la préposition dans.

\section{Le site}

Le domaine du site est circonscrit : il est dénoté par le constituant régi par la préposition. En position initiale, le SP précède l'introduction de la cible et est prédisposé à une portée large sur l'énoncé. D'un point de vu ontologique, il peut s'agir d'une entité qui s'apparente :

- à un lieu ${ }^{12}$ : support (21), institution (22), objet textuel (23), un monde virtuel (24), un domaine (25) ou domaine restreint (26) ;

- à une situation : état (27), événement (28);

- à une qualité ou événement qualitatif (29) ;

- à un objet /un individu (30). 
(21) Dans un communiqué ; Dans son livre ; Dans son bulletin mensuel de décembre publié ; Dans une lettre ouverte rendue publique; Dans un entretien publié par Time Magazine; Dans un communiqué commun; Dans une lettre adressée à M. Seillière; Dans un communiqué publié à Cayenne; Dans le mensuel Défense nationale 2000 ; Dans un entretien exclusif au Monde

(22) Dans le Conseil ; Dans cette foire aux vanités qu'est devenu le Conseil européen

(23) Dans le résumé

(24) Dans Angel; Dans une scène du début; Dans les livres de souvenirs hollywoodiens ; Dans cet imbroglio brillantissime

(25) Dans l'univers ultra évolutif du Net; Dans la catégorie des métaphores hasardeuses ; dans l'ancien slave

(26) Dans les deux tiers des familles interrogées; Dans huit cas sur dix; Dans le cas limite que présente l'affaire controversée... ; Dans le cas du Movatar ; Dans ce cas

(27) Dans leur solitude pourtant accompagnée de multiples moyens de transmission, Dans la crainte de représailles; Dans l'attente d'un règlement de ce contentieux; Dans l'attente de confirmation; Dans ce contexte; Dans l'état actuel des choses ; Dans ces conditions ;

(28) Dans cette course placée sous le signe de la sécurité depuis les trois chavirages de 1995-1996 ; Dans ce raccourci temporel qui cogne deux époques contre ... ; Dans cette espèce; Dans l'affirmative

(29) Dans des tons de guimauve; Dans un crissement imperceptible de soie et de mousseline; Dans une version maximaliste et kitschissime; Dans une vision féerique ; Dans une surenchère démonstrative

(30) Dans son logo de trois atomes tournoyants ; Dans le cas du Slugbot ; Dans le cas de ces armes chimiques ou biologiques appelées ; Dans son cas ; Dans le cinéma

Il faut noter que la catégorisation des entités Site est parfois contextuellement déterminée par le point de vue du locuteur. Par exemple, nous avons rangé Dans le cinéma avec les objets parce qu'il est, dans l'exemple (31), l'objet d'une perception (d'un jugement). Il pourrait être dans d'autres contextes un lieu (réel ou virtuel, un domaine, une institution).

(31) [* ${ }^{*}$ Dans * > le cinéma *], aujourd'hui, je ne vois que les recettes infâmes des success stories hollywoodiennes, ou ailleurs un monde brouillé, plein de regrets, de désespoir ou d'ironique résignation.

Il faut également noter que toutes les catégories peuvent véhiculer des informations qualitatives qui traduisent le point de vue du locuteur. De fait, les SP en position initiale se prêtent massivement à cette fonction en véhiculant à travers une expansion du SN (32) ou sous la forme d'une description définie (33) une vision personnelle, ou une information cruciale $^{13}(34)$, essentielles pour l'établissement de la relation avec la proposition.

(32) $\left[*{ }^{*}\right.$ Dans ${ }^{*}>$ cette foire aux vanités qu'est devenu le Conseil européen $\left.{ }^{*}\right]$, quinze chefs d'Etat ou de gouvernement-pas une femme-se sont livrés à une entreprise insidieuse de démantèlement de l'Europe communautaire.

Dans cet exemple (32), il y a une influence des qualités attribuées au conseil sur ses membres qui semblent en hériter. C'est dans le tour qualifiant que cet emploi est particulier. Il redéfini une institution (le Conseil européen) comme une foire aux vanités. Dans ce "monde révélé", on peut expliquer que les chefs d'Etat puissent démanteler l'Europe communautaire et même on peut établir un lien de causalité.

(33) [* <* Dans *> cet imbroglio brillantissime *], les fenêtres, les portes et le grand escalier 1930 tiennent le haut du pavé comme dans un vaudeville.

Dans cet exemple (33), la description définie cet imbroglio brillantissime dénote le film dont le journaliste vient de faire une critique enthousiaste. L'effet produit, qui est de 
communiquer son enthousiasme jusqu'au rôle que peuvent jouer 'les fenêtres, les portes et le grand escalier 1930', serait annulé s'il avait écrit simplement 'dans ce film'. C'est grâce au filtre que pose l'énonciateur par la description définie en imposant la 'couleur' brillantissime que l'on est amené à voir du 'brillant' dans les portes et les fenêtres.

(34) [* <* Dans *> l'univers ultra évolutif du Net *], la mutation des modèles économiques est non seulement possible, mais quasi requise. «La plupart des net entreprises modifient leur modèle économique trois à quatre fois par an ", explique Patricia Seybold

Enfin, dans l'exemple (34) apparaît clairement le lien de causalité entre le fait, pour le Net, d'être un univers ultra évolutif et la nécessité, pour les modèles économiques, de muter en conséquence. La construction [Dans SN, P] serait bancale si l'on omettait de préciser la qualité 'ultra-évolutif de l'univers en question. La qualité est, dans la construction, une information cruciale sur laquelle repose le lien de cause à effet.

Il est clair que l'on voit se profiler comment la notion de dépendance existentielle proposée par Vandeloise peut se décliner à travers la relation causale, les effets n'étant que le produit des causes.

Nous n'avons pas fait le tour dans cette description du site de toutes les fonctions qu'il peut prendre mais nous verrons cela plus en détail dans la section 3 , après avoir regardé du côté de la cible.

\section{La cible}

La construction [Dans SN, P] ne correspond pas systématiquement à [Dans $y, x]$ où $x$ et $y$ sont la Cible et le Site. Un point frappant de la construction est qu'elle se prête aisément à véhiculer le point de vue du locuteur qui qualifie le $y$ d'une part et le met en relation avec un $x$ d'autre part. Les marques de l'acte de l'énonciation sont généralement effacées.

Le locuteur s'adresse à son interlocuteur en effaçant sa trace comme dans (34) où il faut entendre un: je vous dis que/ on sait que 'la mutation des modèles économiques est non seulement possible, mais quasi requise - dans l'univers du Net qui est ultra évolutif'.

Le $x$, comme le $y$ sont dans la portée du dire pris en charge par le locuteur. Quand, $y$ est détaché en position extraprédicative, il ne porte pas sur l'acte d'énonciation (le dire : *Je dis dans l'univers ... du Net), ni même sur l'énoncé lui-même (le dit : la mutation des modèles économiques est non seulement possible, mais quasi requise), mais sur l'état des choses dépeint par le dit. Ainsi, sont mis en relation $x$ (la nécessité des mutations des modèles économiques) dans $y$ (l'univers ultra évolutif du Net). Ce qui est soulevé ici c'est l'influence qu'exerce la qualité du $y$ sur $x$.

Dans l'exemple (35) ci-dessous,

(35) [* $<^{*}$ Dans ${ }^{*}>$ son entreprise de lobbying $\left.*\right]$, la Norvège, même si elle affirme ne parler qu'en son nom, représente également les intérêts d'un autre intervenant, le Japon, qui s'inquiète des entraves qui pourraient être imposées par l'Europe au libre ...

le locuteur prend de la distance avec son dit pour faire entendre une deuxième voix (' même si elle affirme ne parler qu'en son nom'). Cette deuxième voix, de commentaire en quelque sorte, n'est pas impliquée dans la relation dans. Pour autant, on ne peut dire que dans $S N$ porterait sur le seul sujet la Norvège. Ce qui est mis en relation c'est $y$ (le lobbying exercé par la Norvège) et $x$ (la représentation des intérêts non seulement de la Norvège mais aussi de ceux du Japon). Ainsi l'action de lobbying entreprise par la Norvège englobe 
ses intérêts propres et ceux du Japon mais tendrait à nous cacher si l'on n'y prêtait attention, ce deuxième intervenant intéressé. En outre, ce qu'on peut donc inférer, c'est que l'action visible en $y$, cache un enjeu établi comme $x$.

Nous voyons à travers ces exemples que l'identification de la cible proprement dite nécessite, pour le moins, un certain effort. Pourtant, l'interprétation des constructions [ Dans $S N, P]$ ne nécessite pas d'effort particulier, indépendamment de la grande variation qu'elle présente. Nous ne butons pas dessus pour les comprendre. Quel est donc le principe sur lequel repose notre compréhension immédiate de cette construction? La question peut paraitre naïve car il y a, de fait, toujours ce décalage entre la simplicité apparente de traitement et la complexité d'analyse que le linguiste cherche à élucider. Cependant, elle peut avoir un pouvoir euristique pour nous faire avancer. Entre autre, elle nous amène à suggérer que si la saisie de la relation est immédiate c'est quelle repose sur un principe général qui subsume la variation. Nous gardons cette idée en ligne de mire lors de notre examen, dans le \$2.2.2. ci-dessous, du degré d'adéquation de la relation [Dans $S N, P]$ avec les traits de la ressemblance de famille $\mathrm{C} / \mathrm{c}$.

\subsubsection{La relation $[$ Dans $\mathrm{SN}, \mathrm{P}]$ et la relation $\mathrm{C} / \mathrm{c}$}

\section{(a) Mise en place de la distinction prédication (propriété contingente) vs. attribution (qualité essentielle)}

Examinons un cas pour lequel aucun trait de la ressemblance de famille $\mathrm{C} / \mathrm{c}$ n'est satisfait. Prenons l'exemple (36).

(36) [* <* Dans * $^{*}$ son ensemble *], le JT de la Une a été regardé par 11250000

téléspectateurs, soit $46,5 \%$ de PDA. Les deux soirs précédant l'entretien avec M.

Chirac, le journal télévisé de TF1 avait réalisé des PDA respectives de 39,3\% et de $39,4 \%$

Si le SP était intégré à la proposition il dépendrait clairement du procès : regarder le JT dans son ensemble. On pourrait y opposer regarder le JT en partie seulement. Dans cette position, le SP précise les bornes du procès en ajustant le temps du procès (être regardé) sur la durée de l'événement audio visuel (le JT). Le procès dure autant que l'événement se déroule.

Déplacé en tête, détaché de la prédication, il semble perdre son pouvoir de quantification de la durée du procès. D’ailleurs, on pourrait difficilement avoir (36') :

(36') ? ? En partie/ En partie seulement, le JT de la Une a été regardé par 11250000

téléspectateurs,

En outre, on perçoit bien dans cet exemple (que le SP soit intra ou extraprédicatif) que, comme dans les rapports portion/masse et membre/classe, la relation d'inclusion 'est complètement brisée' (Vandeloise $2001: 255$ ).

De même qu'on ne peut dire *la portion de melon est dans le melon, ou * les moineaux sont dans les oiseaux, on ne peut dire* le JT est dans son ensemble comme si dans n'était plus prédicatif (prédication d'une propriété) mais attributif (attribution d'une qualité). Dans $y$, devient une qualité essentielle de $\mathrm{x}$, constitutive de $\mathrm{x}$, et non plus contingente ou accidentelle. Ainsi le JT dans son ensemble s'apparente à l'ensemble du JT. Quelle est cependant la différence entre les deux tours? Le SP Dans son ensemble dénote-t-il vraiment une qualité essentielle ? Il semble que dans laisse la place, de par son détachement par rapport à $x$ (le JT), à une possible saisie extérieure de la relation attributive qui peut ainsi être modulée par le locuteur au gré de ses points de vues (Dans presque tout son ensemble, le JT...) ${ }^{14}$. Ainsi 
dans $y, x$ permettrait d'instaurer une relation attributive (qualifiante de $x$ ) et subjective, en la faisant passer pour constitutive de $x$.

Cette disjonction entre emplois prédicatifs et attributifs est indépendante de la forme qui l'exprime. Si on dit le vin dans le verre en omettant le prédicat/ copule (être), on n'obtient pas pour autant l'interprétation attributive (qui pourrait être exprimée par un verre de vin où le vin est constitutif du verre de vin). L'interprétation attributive résulte pour dans de l'impossibilité ontologique pour $y$ de contenir $x$. C'est le cas lorsque $y$ (le Site) est constitutif d'une quelconque façon de $x$ (la Cible) ce qui correspond à une conception opposée de l'inclusion de $x$ dans $y$.

Cette intrusion de $y$ sur l'être de $x$ présente une variation graduelle d'intensité. Nous allons à présent pouvoir poursuivre notre examen de l'adéquation de la relation [Dans SN, $\mathrm{P}]$ aux traits de la relation de famille $\mathrm{C} / \mathrm{c}$ à la lumière de ce continuum où $y$ interpénètre plus où moins la sphère essentielle de $x$.

\section{(b) Scores des traits de la ressemblance de famille $c / c$}

Sur l'ensemble des emplois notionnels considérés, dans la construction [Dans SN, P], les traits Dépendance (c dépend de $\mathrm{C}$ ) et Influence ( $\mathrm{C}$ influence $\mathrm{c}$ ) ont un score de 35 et $37 \%$ respectivement. Ils sont massivement employés au détriment des autres traits. (voir détails, figure 4)

Figure 4

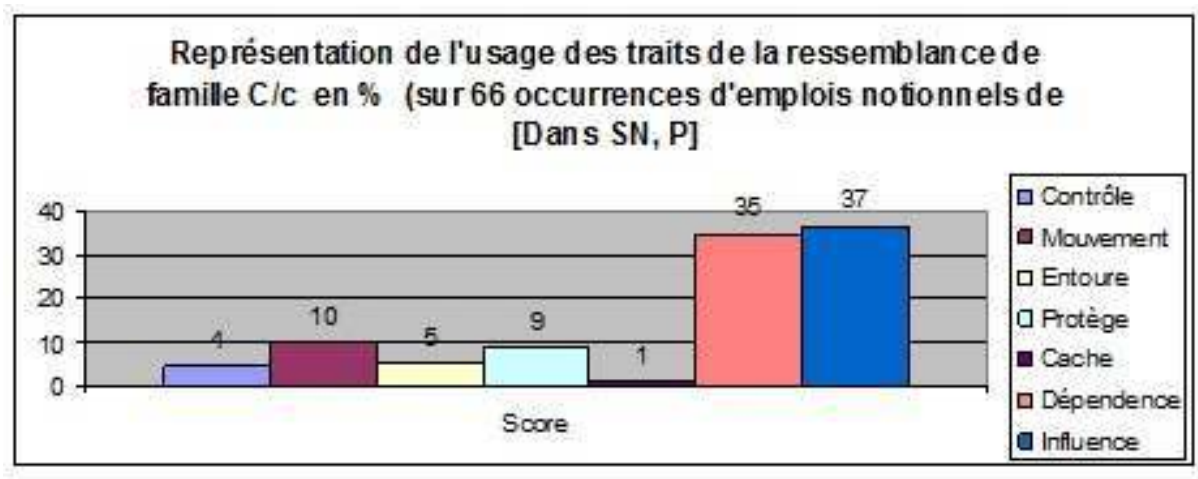

La figure 5 ci-dessous montre que dans plus de la moitié des cas (53\%), un seul trait est satisfait ; dans $(28 \%)$, deux traits.

Figure 5

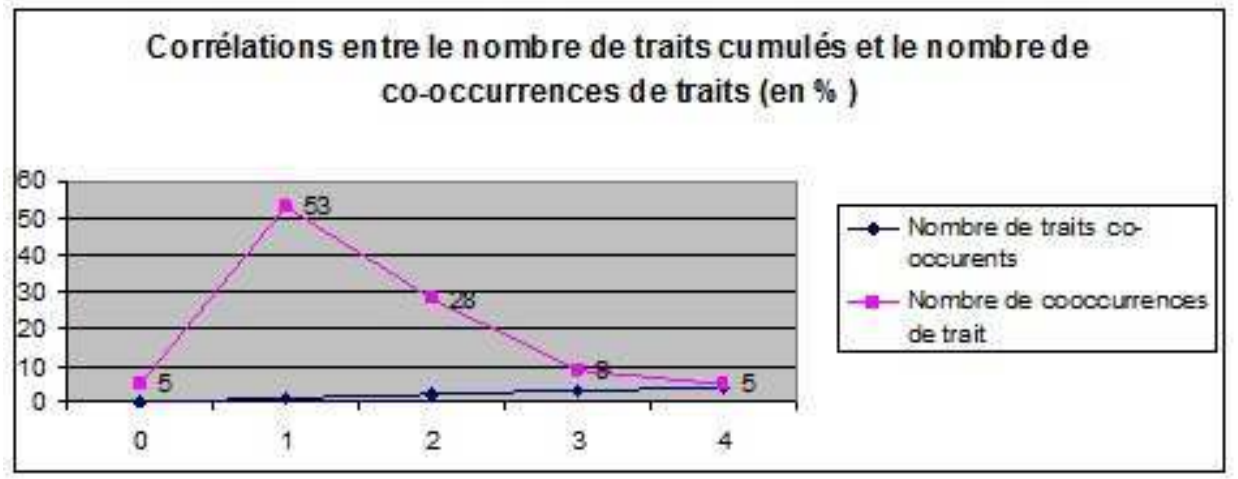


Partant, il apparaît clairement que les traits Dépendance et Influence sont essentiels pour saisir ces emplois notionnels dans la construction [Dans SN, P], et sont souvent les seuls traits que l'on puisse appliquer.

\section{Illustration des modes d'interpénétration de $x$ et $y$ dans la construction [Dans SN, P]}

Nous voulons dans cette dernière section proposer un chemin pour organiser les emplois notionnels que nous avons rencontrés dans la construction [Dans (SN), P]. Nous proposons de les relier les uns aux autres sur un continuum allant d'un pôle localisant $x$ au pôle opposé définitoire de $x$ (i.e. des emplois prédicatifs aux emplois attributifs). On peut donner une idée de cette opposition en concevant une inversion progressive des rôles typiques de la cible $(x)$ et du site $(y)$. Au pôle localisant, la relation $\mathrm{C} / \mathrm{c}$ est à son comble ${ }^{15}$, le contenu est localisé par le contenant (ex. Dans un communiqué, ...). Au pôle définitoire, le contenant recatégorise le contenu.

49 A ce continuum du localisant au définitoire sont corrélées trois dimensions associées, celle de la causalité, de la subjectivité ${ }^{16}$ et d'une forme de dynamicité ${ }^{17}$. Plus on est au pôle localisant, plus la relation est objective et moins elle est causale. Plus on va vers le pôle définitoire, plus le point de vue du locuteur s'exprime, dans la tournure qualifiante et plus la relation devient causale.

Entre ces deux pôles, l'ordonnancement des différents 'sens' n'est pas tâche facile. Nous faisons une première proposition avec cependant la conscience aigue de la nécessité d'étudier minutieusement chaque cas, la nécessité de mettre en place une série de tests et de transformations possibles permettant d'évaluer finement leur positionnement sur ce continuum, ce qui laisse encore un beau programme de recherche devant nous.

En attendant, notre proposition spéculative est la suivante :

Figure 6

\begin{tabular}{|l|l|l|l|l|l|}
\hline $\begin{array}{l}\text { Y } \\
\text { localise> }\end{array}$ & $\begin{array}{l}\text { fixe un domaine } \\
\text { d'interprétation> }\end{array}$ & $\begin{array}{l}\text { pose une } \\
\text { condition> }\end{array}$ & topicalise> & qualifie> & définit X \\
\hline - causal & & & & & + causal \\
\hline + objectif & & & & & + subjectif \\
\hline + statif & & & & & + \\
\hline
\end{tabular}

Continuum du localisant au définitoire

Dans la suite de cette section, nous passons rapidement en revue chaque cas. 


\subsection{Localisation : $y$ repère, véhicule, conserve, donne à voir / entendre $x$}

51 Dans cette configuration, nous sommes au plus proche possible, pour les emplois notionnels, de la relation $\mathrm{C} / \mathrm{c}$. Ce sont ces emplois qui cumulent le plus de traits. Prenons l'exemple (37) :

(37) [* ${ }^{*}$ Dans * $>$ une scène du début *], un petit figurant mime Hitler, rôle muet, il

se rajoute une phrase au moment du salut, « Heil moi-même ».

Cet exemple décrit une scène d'un monde fictif (film), mais pour autant représentée comme une scène du monde réel. La scène en question est localisée spatiotemporellement par $y$ (Dans une scène du début) dans une des parties constitutives du film qui se situe au début. Par le biais du SP, elle est ancrée dans le monde fictif. Il faut donc abstraire la représentation de la cible qui s'exprime sous la forme d'une description (un petit figurant etc.) en une partie d'une scène (la séquence où un petit figurant...) pour la resituer dans une scène du début. Cette capacité à ainsi saisir la description qui est faite comme une séquence d'une scène nous est donnée via son ancrage en tête par le SP(Dans une scène du début) dans le monde de la fiction. Dans pose ici une relation entre une scène ( $y$ ) et une partie de cette scène $(x)$.

53 L'analogie avec l'ancrage d'une scène réelle dans un monde réel est presque complète à ceci près que dans le monde fictif les entités manipulées par la relation dans entretiennent ici une relation composant/assemblage (une séquence de la scène du début) ou de façon moins technique une relation morceau/tout (un passage de la scène du début) ${ }^{18}$. Si on conceptualise la relation d'une séquence dans un film, on peut établir ${ }^{19}$, a) que la scène contrôle la position de la séquence qui a été fixée lors du montage; b) que le trait mouvement de $\mathrm{c}$ vers $\mathrm{C}$ est difficilement extensible dans ce cas ; c) que l'on peut concevoir que la scène entoure et même inclut la séquence ${ }^{20}, d$ ) que le trait ' $C$ protège $c$ ' est difficilement extensible à ce cas, e) qu'il en est de même du trait ' $C$ cache $c$ '; f) que la possibilité d'analyser une scène en séquence dépend de l'existence matérielle de la scène ; et g) que l'interprétation de $\mathrm{P}$ comme séquence constitutive d'une scène du début résulte du domaine que définit le SP en dans. On pourrait dire que Dans d'une scène du début exerce une influence sur l'interprétation de $\mathrm{P}$ comme séquence. Nous avons là quatre traits satisfaits : contrôle, entoure, dépendance et influence.

\subsection{Domaine d'interprétation : $y$ délimite le domaine d'interprétation de $x$}

54 C'est le rôle typique des compléments détachés qui déterminent l'univers ou le domaine dans lequel P sera vraie (Martin 1983, Charolles 1997). A la suite de Charolles, on les appelle des cadres de discours, parce qu'en position initiale, ils sont à même d'indexer un segment au-delà de leur proposition d'accueil et d'intégrer une masse d'informations pour lesquelles ils fixent un critère d'interprétation. Pour les SP spatiaux, par exemple, leur fonction est de délimiter une zone $y$ où $x$ peut être localisé. Cette fonction se cumule avec cette autre fonction d'index, qui est de créer par là-même un domaine de validité pour $P$ (et au-delà). Les SP qui remplissent cette fonction arrivent souvent en série dans le discours mettant en jeu des contrastes où des parallélismes (Dans les chaumières, ... Dans les 
palaces, ...). Dans le domaine notionnel ${ }^{21}$, on trouve surtout des cadres praxéologiques (cadres de domaine) comme en (38) ou (39), mais aussi de localisation (40) :

(38) [* <* Dans *> l'ancien slave *] (slave venant, au fait, du latin slavus, esclave), le mot rabota signifie justement « esclavage ».

(39) [* <* Dans *> les champagnes millésimés *]-les meilleures années uniquement-, aucun vin antérieur à l'année de récolte ne peut être ajouté.

(40)[* <* Dans * les livres de souvenirs hollywoodiens *], Lubitsch apparait de ci, de là, comme un génie bienfaisant, réconfortant Ingrid Bergman au lendemain de son arrivée en Californie, enseignant à Billy Wilder les arcanes de la mécanique et du rythme (...)

En (41), on voit que dans le cas du Movatar crée un contraste en se différentiant du cas introduit plus avant par Normalement :

(41) Normalement, l'avatar se substitue à l'humain pour naviguer dans l'espace virtuel des réseaux et explorer le cybermonde. [* ${ }^{*}$ Dans * le cas du «movatar *] », l'avatar animé par fonctions algorithmiques vient squatter le corps de Stelarc pour explorer le monde réel.

\subsection{Condition : $y$ est la protase pour $\mathrm{x}$ l'apodose}

On peut tenter de voir comment on peut passer d'une fonction cadrative du SP (comme nous venons de le voir cf. 4.2), à une condition en restreignant le domaine d'interprétation de P. Trouveraient leur place ici des exemples comme (42-44)

(42) [* <* Dans *> l'affirmative *], certains n'excluent pas que cette nouvelle réunion pourrait se tenir très rapidement, peut-être dès la semaine prochaine.

(43) $\left[*{ }^{*}\right.$ Dans $*>$ certaines conditions encore très mal connues $\left.*\right]$, le virus se réveille à l'intérieur du neurone.

(44) «Suivant le terrain où il devra évoluer, on peut, par exemple, déterminer si le nombre de pattes optimal est quatre ou six ", poursuit Jean-Arcady Meyer. [* <* Dans $*$ > ce cas*], des simulations sont faites sur ordinateur jusqu'à l'obtention de la configuration optimale.

En (42), Dans l'affirmative est équivalent à dans l'hypothèse d'une réponse positive. Cette hypothèse est réellement posée et déclenche l'usage du conditionnel dans l'apodose. En (43) et (44), nous avons au contraire un présent ou un passif. Le SP en (43) est un générique sous spécifié pour un ensemble de conditions (mal connues). Mais si on a une de ces conditions, alors P (le virus se réveille). Dans l'exemple (44), on peut voir une illustration soit du fait que le journaliste, auteur de ce texte, n'écrit pas de la plus belle manière, soit du fait que cas et un marqueur flottant qui oscille entre la localisation, la condition et la topicalisation. A travers la variation des exemples [dans () cas (),], nous avons pu observé toutes ces nuances sans bien les cerner encore. En (44), dans ce cas pourrait fonctionner comme simple anaphorique en reprenant une des deux options évoquées : un robot à quatre pattes ou un robot à six pattes. Et on centrerait ainsi l'attention sur une de ces deux options. Mais on est dans l'obligation au vu de la suite, d'interpréter dans ce cas, comme le cas où on souhaiterait déterminer quel est le nombre de pattes optimal et il s'agit alors bien d'une condition : si l'on veut obtenir le nombre de pattes optimal alors on fait des simulations.

Pour ce type d'exemples qui expriment une condition, le trait essentiel est la dépendance de $x$ par rapport à $y$. 


\subsection{Topicalisation : $y$ devient ce dont $x$ est à propos}

57 On bascule de la condition à la topicalisation en considérant le SP pour lui-même, et non comme membre d'une alternative. La phrase $\mathrm{P}$ qu'il introduit est une affirmation, une déclaration.

(45) $\left[*<^{*}\right.$ Dans * $>$ son cas $\left.{ }^{*}\right]$, c'est inutile : il ne pourrait obtenir que trois points, alors que s'il ne commet aucune autre infraction pendant trois ans, son capital sera automatiquement reconstitué.

En (45), il n'est plus question de considérer son cas parmi d'autres. Nous ne sommes plus face à si on considère son cas, alors, mais plutôt face à En ce qui le concerne, Quant à lui etc., on affirme $x$ qui le concerne. Quelle est donc la relation de $x$ et $y$ dans cette construction où le SP en dans est marqueur de topicalisation? Pour reprendre l'image de l'inversion progressive des rôles de cible et de site sur le continuum entre les pôles localisant et définitoire, la topicalisation serait sans doute le point d'intersection (le point d'équilibre) où les rapports s'inversent. Avant que la dynamique ne s'engage dans la direction de la qualité, avant que le $y$ ne soit partie prenante du $x$. On peut les voir mis côte à côte l'un de l'autre sans qu'aucune force ne soit exercée dans un sens ou dans l'autre. Leur mode de mise en relation serait l'association via la juxtaposition. Les exemples (46-47) sont d'autres illustrations de ce rapport de juxtaposition :

(46) [* $<^{*}$ Dans * $>$ le cas de l'Argentine *], le Fonds a bien accepté de desserrer l'étau d'un objectif de déficit budgétaire impossible à tenir et a accepté qu'il soit porté de 4 à 6 milliards pour 2001

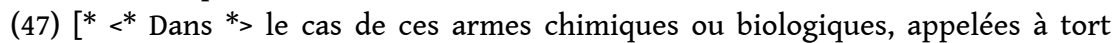
« armes du pauvre » alors même que la bombe atomique a cessé d'être l'exclusivité des riches*], il est un pays dont la politique singulière est fort préoccupante (...)

\subsection{Qualification : $y$ devient une qualité qui s'incorpore en $x$, voire qui définit $x$}

Pour illustrer cette classe d'usages que nous avons déjà bien circonscrite en 2.3. je prendrai deux derniers exemples pour montrer comment [Dans SN, P] dans ses usages attributifs permet à la fois d'attribuer des qualités et de définir/catégoriser $x$.

Quand dans $y$ attribue une qualité, comme en (48), la relation $\mathrm{C} / \mathrm{c}$ ne tient plus qu'à un fil (la Dépendance de $x$ par rapport à $y^{22}$ ), le contenant devient consubstantiel du contenu, il est transmuté en qualité et le contenu en réceptacle de cette qualité (ex. Dans la crainte des représailles..., $\rightarrow$ les organisations sont pleines/ remplie de la crainte des représailles). Ce qui était l'entité à localiser devient l'entité localisatrice d'une qualité qui le submerge et détermine, cause sa façon d'agir.

(48)[* ${ }^{*}<^{*}$ Dans ${ }^{*}>$ la crainte de représailles *], non seulement l'ONU, mais aussi les organisations non gouvernementales, ont déjà procédé à l'évacuation de leur personnel humanitaire en Afghanistan.

(49) [* ${ }^{*}$ Dans * ${ }^{*}$ une décision historique *], le magistrat a inculpé l'ancien homme fort du Chili comme « auteur intellectuel » des crimes commis par la « caravane de la mort».

61 En (49) le SP (Dans une décision historique) définit, à travers son adjectif, la qualité de l'inculpation qui est historique. Dans permet de connecter dynamiquement une qualité à un événement. Et à travers le nom (une décision) le SP recatégorise tout l'événement en 
une décision historique si bien qu'on pourrait paraphraser l'extrait par: l'inculpation de l'ancien homme fort du Chili par le magistrat fut/ est une décision historique.

Nous ne sommes plus dans le domaine de la localisation mais dans le domaine de l'être. La décision et l'inculpation sont consubstantielles. Aucun trait de la ressemblance de famille ne caractérise cet usage.

\section{Conclusion}

Dans cet article, nous avons voulu rendre hommage à Claude Vandeloise qui continue d'éclairer nos raisonnements. Nous avons tâché de rendre hommage à sa véritable âme de chercheur qui est allé explorer jusqu'aux particules de la matière pour mieux saisir comment le langage s'en empare.

En suivant la piste qu'il avait tracée en élargissant la relation $\mathrm{C} / \mathrm{c}$ organisée en ressemblance de famille à deux nouveaux traits (Dépendence) et (Influence), nous avons exploré dans la construction [Dans SN, P] les usages dits notionnels de dans. Notre étude, basée sur corpus, contient les limites du corpus. Cependant, à travers les occurrences examinées nous avons mis au jour un principe assez simple pour expliquer et connecter entre eux les différents sens notionnels que nous avons relevés. Nous avons proposé de les organiser sur un continuum entre un pôle localisant et un pôle définitoire. Nous avons suggéré que le passage d'un pôle à l'autre s'accompagne d'une inversion des rôles de Contenant et de contenu (cf. Dans la crainte des représailles, $x$ ), voire à une con-fusion totale des rôles (Dans une décision historique, $x$ ) lorsque Dans $y$ revient, en fin de compte, à recatégoriser $x$. De plus nous avons associé deux dimensions à ce continuum: la dimension de la causalité et celle de la subjectivité. Nous avons suggéré qu'en allant vers le pôle définitoire la causalité et la subjectivité augmentaient. Enfin, nous imputons cet effet au moins en partie à la construction détachée elle-même, qui par le truchement du détachement laisse la place à la voix du locuteur d'exprimer son point de vue.

Beaucoup de points n'ont été qu'évoqués, à partir de cette exploration première, nous avons conscience de l'ampleur du chemin qu'il reste encore à défricher.

\section{BIBLIOGRAPHIE}

Aurnague, M. 2004. Les structures de l'espace linguistique : regards croisés sur quelques constructions spatiales du basque et du français, Peeters (Bibliothèque de l'Information Grammaticale 56).

Bourigault, D. 2007. Un analyseur syntaxique opérationnel : SYNTEX, Thèse d'habilitation à diriger des recherches, Université de Toulouse le Mirail.

Charolles, M. 1997. «L'encadrement du discours : univers, champs, domaines et espaces ». Cahier de recherche linguistique 6. Nancy : Université Nancy $2:$ 1-73. 
Enkvist, N. E. 1989. « Connexity, Interpretability, Universes of Discourse, and Text Worlds ». In J. Allen (ed) Possible Worlds in Humanities, Arts and Sciences. Berlin/New-York: Walter de Gruyter, 162-186.

Evans, V., \& Tyler, A. 2004. "Spatial experience, lexical structure and motivation: the case of in ", In G. Radden \& G. Panther (Eds.), Studies in Linguistics Motivation [In The Cognitive Linguistics Research Series]. New York and Berlin: Mouton de Gruyter: 157-192.

Franckel, J-J. \& Paillard, D. 2007. Grammaire des prépositions, Tome I, Paris : Ophrys.

Hopper, P. J., \& Traugott, E. C. 2003. Grammaticalization (2nd ed.). Cambridge University Press.

Martin R., 1983. La logique du sens, Paris : PUF.

Lakoff, G.. 1987.Women, fire, and dangerous things: What categories reveal about the mind. Chicago : University of Chicago.

Leeman, D. (1994). « Dans et les noms animés », Cahiers de grammaire, 19, Toulouse, Université de Toulouse-Le Mirail, ERSS (pp. 19-33).

Leeman, D. (1999). «Dans un juron, il sauta sur ses pistolets : aspects de la polysémie de la préposition dans ", Revue de Sémantique et Pragmatique, 6, Orléans, Presses Universitaires d'Orléans (pp. 71-88).

Leeman, D. (2008). Langue française $\mathrm{n}^{\circ} 157$ : «Énigmatiques prépositions », Paris, Larousse.

Stewart, J. (2001). « La conscience en tant que métaphore spatiale : la théorie de Jaynes. » Intellectica, 2001/1, $32: 87-110$.

Vandeloise, C. (1986). L'espace en français. Sémantique des prépositions spatiales. Le Seuil, Paris. Vandeloise, C. (2001). Aristote et le lexique de l'espace - rencontres entre la physique grecque et la linguistique cognitive, Stanford, Éditions CSLI.

Vigier, D. (2008). «Contribution à une étude des constructions antéposées du type : En homme intelligent et humain, il partagea tout de suite l'inquiétude de Marcel (J. Verne) », Discours, 2 | 2008, [En ligne], mis URL : http://discours.revues.org/index863.html. Consulté le 27 octobre 2009.

Tyler A. \& Evans V. 2003. The Semantics of English Prepositions: Spatial Scenes, Embodied Meaning, and Cognition. Cambridge University Press.

\section{NOTES}

1. Voir entre autre Vandeloise 1986, 1993.

2. Vandeloise introduit cette définition « a est dans b si b entoure a " parce qu'elle vaut pour tout type d'entité, matérielle et spatiales. Alors que la définition (topologique) « a est dans b si b inclut $a$ » ne vaut que pour des entités spatiales, sinon il est besoin de faire appel à la place extension de a et b. (Vandeloise 2001 :227).

3. Cette remarque est contredite par un exemple qu'il donne à la page précédente : le cadeau est dans l'emballage. Il note que si, dans cet exemple, le trait (a) n'est pas satisfait (c'est la position du cadeau qui contrôle celle de l'emballage et non l'inverse), les traits (d) (l'emballage protège le cadeau) et (e) (l'emballage cache le cadeau) sont satisfaits.

4. Il s'agit des exemples le trou est dans la table, l'oiseau est dans le ciel et La Corse est dans la Méditerranée qui illustrent respectivement trois types de situations: (a) cible spatiale/ site matériel, (b) cible matérielle/ site spatial, et (c) cible spatiale/ site spatial.

5. (Cf. Bourigault 2007). 
6. Ce projet dirigé par Michel Charolles et Laure Sarda a été financé par l'ANR (2006-2009). La constituée permet d'établir les corrélations entre le sens et la position de SP introduits par 9 prépositions différentes (à, dans, sur, en, depuis, par, à travers, jusque, vers). Elle contient plus de 16000 SP annotés.

7. Le SP peut être en queue s'il est à la fois régi par un verbe et le dernier constituant de la phrase. Cette position que nous appelons, médiane correspond à une fonction argumentale, les positions périphériques tête et queue (non régies) correspondent à des fonctions adverbiales, où à des prédications secondes.

8. Les rattachements syntaxiques effectués automatiquement par SYNTEX reposent sur une analyse statistique sur un grand corpus (10 ans du Monde). Les résultats comprennent un taux d'erreur, plus important en zone post-verbale dont il faut avoir conscience.

9. Le test pour appliquer ce trait est la possibilité d'appliquer la paraphrase : Locuteur V(dire) dans Y que X (où X est du discours rapporté)., comme dans l'exemple (11) paraphrasable par « la banque [dit/juge/ estime/ déclare/annonce...] dans son bulletin qu'il est souhaitable que... ».

10. Cette valeur a été proposée par Denis Vigier pour décrire des emplois spécifiques de SP régis par la préposition en et dénotant un déguisement. De l'exemple que nous lui empruntons (Vigier 2008 : §4), Au bal masqué de Luc, Marie est arrivée en nonne on peut déduire qu'elle était en nonne. Ces cas ne doivent pas être assimilés aux cas où le SP en en introduit un $N$ dénotant une attitude, un comportement, un statut, une propriété plus ou moins permanente d'un actant: Marie a réglé cette affaire en femme de tête. *Elle était en femme de tête. Cependant la frontière entre les deux n'est pas toujours facile à tracer et c'est une piste que nous suivrons dans notre examen des SP en dans.

11. Le but, qui n'est pas représenté en tête ne sera pas examiné ici.

12. Nous adaptons la définition d'Aurnague 2004. Nous considérons le lieu dans une acception large, libéré de la composante matérielle, au sens d'une portion d'espace qui constitue un cadre de référence fixe pour une cible. Ceci afin de pouvoir intégrer les mondes virtuels et conceptuels. 13. Nous reprenons le terme à Enkvist (1989) qui a défini le principe CIF (Crucial Information First). Information que l'énonciateur juge cruciale dans l'établissement de la relation du SP en dans et la proposition qu'il introduit.

14. On pourrait capter par ce biais, l'emploi paradoxal de dans l'ensemble qui signifie en somme en grande partie. Dans l'exemple Dans l'ensemble, les enfants ont été sages, le SP dans l'ensemble 'absorbe' les 'moments' où les enfants n'ont pas été sages pour, dans une sorte de moyenne, assimiler l'ensemble à un état de sagesse.

15. Pour autant qu'elle puisse y être dans le domaine notionnel, disons qu'à ce niveau on reste dans un espace métaphorique où une entité (une déclaration) est localisée dans une autre (un communiqué de presse).

16. Ces dimensions pourraient être imputées directement à la construction, au fait que dans le détachement se crée l'espace par lequel le locuteur peut faire entendre sa propre voix, et la partition entre la cause et son effet qui reflèterait son intention communicative.

17. Il faut comprendre dynamicité dans le sens où la mise en relation de Dans $y$, avec $x$ est dynamique. Dans le cas d'un Dans y localisant, le Sp denote un cadre de référence qui est posé (statique) pour l'interprétation de P. On dira que la relation est statique. En revanche, avec le cas d'un Dans y qualifiant ou définitoire, la saisie de la relation est dynamique. Dans y n'est pas posé, mais s'interprète à la lumière de $\mathrm{P}$. Cette question de dynamicité n'a donc rien à voir ici avec la sémantique lexicale ni de la préposition, ni des procès.

18. Dans le monde réel, on saisirait la scène pour ce qu'elle est (un homme mimant Hitler), et on la localiserait dans un lieu (par ex. Dans le centre du village). On aurait donc une relation entre un objet et un lieu.

19. En s'accordant toutefois un peu de liberté sur la définition des traits de la ressemblance de famille $\mathrm{C} / \mathrm{c}$ pour les projeter dans un espace métaphorique.

20. Tous les éléments de la séquence sont des éléments de la scène. 
21. "Dans le domaine notionnel», est un bon exemple de cadre dit praxéologique (fixe le domaine d'interprétation de P).

22. Au sens où $y$ devient une qualité qui s'incorpore à $x$ et devient constitutif de son être.

\section{RÉSUMÉS}

Nous esquissons dans cet article une description des sens notionnels de dans, dans la construction [Dans $\mathrm{SN}_{\mathrm{y}}, \mathrm{P}_{\mathrm{x}}$ ] où le site $\mathrm{y}$ est dénoté par le $\mathrm{SP}$ extraprédicatif [Dans $\mathrm{SN}$ ] et la cible par tout ou partie de la phrase P. Nous observons comment les emplois notionnels de dans, dans cette construction détachée, peuvent être décrits par la relation Contenant/ contenu proposée par Vandeloise. Notre étude, basée sur corpus, propose une classification des différents sens de [Dans $\left.\mathrm{SN}_{\mathrm{y}}, \mathrm{P}_{\mathrm{x}}\right]$ sur un continuum entre un pôle localisant et un pôle définitoire. Trois dimensions sont associées à ce continuum : la causalité, la subjectivité et la dynamicité de la relation entre $y$ et $x$. Vers le pôle définitoire la causalité, la subjectivité et le rapport dynamique entre $\mathrm{x}$ et $\mathrm{y}$ augmentent. Cet effet serait au moins en partie dû à la construction détachée elle-même, qui par le truchement du détachement laisse la place à la voix du locuteur d'exprimer son point de vue.

This paper provides a description of the notional meanings of the French preposition dans ('in') in the construction [Dans $\mathrm{NP}_{\mathrm{y}}, \mathrm{S}_{\mathrm{x}}$ ], where the Ground $\mathrm{y}$ is expressed by the extra-predicative PP [ Dans NP], and the Figure $\mathrm{x}$ by a part or by the whole sentence $\mathrm{S}$. We explore the adequateness of the relation Container/content described by Vandeloise to depict the meanings of dans in this construction. This corpus based study suggests ranging these different meanings along a continuum from a locating pole to a defining pole. Three dimensions are associated to this continuum: the cause, the subjectivity and the dynamicity of the relationship of $y$ and $\mathrm{x}$. On the side of the defining pole, the causality, the subjectivity and the dynamicity of the relationship of $\mathrm{y}$ and $\mathrm{x}$ tend to be more expressed. This could be partly due to the detached construction itself, which through the detachment gives the opportunity to the speaker/writer to express his/her point of view.

\section{INDEX}

Mots-clés : préposition dans ; construction détachée, position initiale, emplois notionnels, sémantique

Keywords : preposition dans ('in'); detached-construction; initial positioning; notional uses; semantics

\section{AUTEUR}

LAURE SARDA

Lattice UMR 8094 CNRS \& ENS 